\title{
Sportverletzungen an der Wirbelsäule
}

\author{
$\square$ Oliver Gonschorek, Patrizia Merkel, Michael Maier, Stefan Hauck, Volker Bühren
}

\section{Zusammenfassung}

Die zunehmende Bedeutung von Wirbelsäulenverletzungen beruht zum einen auf dem gehäuften Auftreten von osteoporotischen Frakturen bei älteren Patienten, wobei Bagatellmechanismen im Vordergrund stehen, zum anderen auf Hochrasanztraumen bei jungen Patienten, wobei Verkehrsunfälle, Stürze aus der Höhe und eben Sport als Ursachen überwiegen. Die Entwicklung der Freizeitgesellschaft mit einem zunehmenden Angebot an Hochrasanzsportarten steuert hierzu einen großen Anteil bei. Die Behandlung von Sportlern mit Wirbelsäulenverletzungen stellt besondere Ansprüche an die Versorgungsqualität im Hinblick auf die Wiedererlangung der sportlichen Leistungsfähigkeit. Entsprechend können die modernen operativen Therapieverfahren besonders vorteilhaft zum Einsatz kommen. Mini- malinvasive endoskopisch gestützte Techniken und moderne Implantate wie z.B. expandierbare Cages ermöglichen heute die konsequente belastungsstabile Rekonstruktion der vorderen Säule bei gleichzeitig deutlich reduzierter Morbidität.

\section{Sports-Related Injuries of the Spinal Column}

Together with osteoporosis and traffic accidents, sports-related injuries play a major role in the onset of spinal complaints. The management of patients with sports-related injuries of the spine is a challenging issue in regard to the ability to resume former sportive activity. A large variety and growing number of high velocity sports lead to a significant rise in spine injuries as well as in neurological lesions. Modern treatment options using minimally invasive techniques such as endoscopic assistance and high tech implants (e.g. expandible cages) show advantageous results in combination with less morbidity. In a two-year-period, 96 patients with sports-related injuries of the thoracic and lumbar spine were prospectively recorded. Conservative (19\%) or operative treatment $(81 \%)$ was performed depending on the extent of the severity and instability of the trauma. In $91 \%$ of the cases, the patients resumed sports participation, only $9 \%$ of the patients had to abandon all sportive activity mostly due to neurological deficits. Minor loss of correction was found in patients with $360^{\circ}$ short-segment fusions, major loss was found after conservative treatment. In conclusion we were able to show that the actual management of spinal injuries effectuates a high rate of resumption of sports activity following conservative or operative treatment.

\section{Einleitung}

Neben Verkehrsunfall und Osteoporose steht die Sportverletzung ganz vorne in der Häufigkeit als Ursache des Auftretens von Verletzungen des thorakolumbalen Übergangs und der LWS, wobei der Wintersport häufig in den Vordergrund gestellt wird $[1,6]$. Während beim älteren Menschen die Osteoporose kausal im Vordergrund steht und bereits Bagatellstürze zu behandlungsbedürftigen Frakturen führen, steht im Sport das Hochrasanztrauma im Vordergrund. Die zunehmende Beliebtheit von Hochrasanz- und Risikosportarten trägt dazu bei, die Bedeutung der Wirbelsäulenverletzung beim Sport stark ansteigen zu

OP-JOURNAL 2008; 24: 158-162

(c) Georg Thieme Verlag KG Stuttgart • New York DOI 10.1055/s-2008-1038961 lassen. Die konservativen und operativen Therapieoptionen sind beim Sportler prinzipiell die gleichen wie bei der übrigen Bevölkerung, allerdings steht die möglichst rasche und vollständige Wiederherstellung bei den meist jungen Patienten noch mehr im Vordergrund, sodass die Tendenz etwas mehr zum operativen Vorgehen hin geht. Bedauerlicherweise nimmt mit der Entwicklung der Risikosportarten auch der Anteil der Hochrasanztraumen und damit auch die Verletzungsschwere zu. Entsprechend ist ein höherer Anteil von Querschnittssyndromen zu beklagen.

Bei der Berichterstattung über Sportunfälle steht gerade auch aufgrund seiner Popularität der Skisport im Vordergrund. So wird in den USA über eine jährliche konsequente Zunahme von schweren Wintersportunfällen (Ski- und Snowboard) berichtet [12,13]. Snow- board und Carving-Ski werden in besonderem Maße angeschuldigt, wobei dies in Europa und hier gerade in Bezug auf Brust- und Lendenwirbelsäulenverletzungen nicht immer mit harten Zahlen belegt werden kann. Steigend sind allerdings die Zahlen bez. der operativen Maßnahmen und hier insbesondere bez. der Rekonstruktion der vorderen Säule. Auch im Rahmen der Untersuchungen der AG Wirbelsäule der DGU wird vermehrt auf die Bedeutung der vorderen Säule hingewiesen [8].

Die Zunahme von schwereren und damit instabilen Verletzungsformen bedürfen in der Regel eines operativen Eingriffs zur Wiederherstellung der Stabilität und der Belastungsfähigkeit der Wirbelsäule. 
Der sportlich aktive Mensch stellt naturgemäß einen hohen Anspruch an seine Genesung und die Wiederaufnahme seiner sportlichen Tätigkeit, entsprechend wird die Indikation zur operativen Therapie eher großzügig gestellt. Dennoch stehen zunächst einmal dieselben Indikationskriterien im Vordergrund, wobei die konservative Behandlung von stabilen Frakturen heute eine frühestmögliche funktionelle Nachbehandlung vorsieht.

Grundlage der differenzierten Versorgung von Wirbelsäulenverletzungen ist die Einteilung in verschiedene Schweregrade der Verletzung und die daraus abzuleitende Instabilität, die üblicherweise nach der AO-Klassifikation erfolgt. Dabei werden instabile Verletzungen durch den stabilisierenden operativen Eingriff (isoliert dorsal, isoliert ventral oder dorsoventral) in eine stabile Form überführt, die eine sofortige frühfunktionelle Weiterbehandlung ermöglicht. Im Weiteren sollen die heutigen operativen Strategien dargelegt und anhand von Fallbeispielen an HWS, BWS und LWS illustriert werden.

\section{HWS}

Hochrasanztraumen führen in hohem Prozentsatz zu Mehrfachverletzungen, der Algorithmus sieht heute für das Polytraumamanagement die primäre Diagnostik des Achsenskeletts mittels Spiral-CT vor. Gefügeverschiebungen und Frakturen der HWS können so insbesondere auch im zervikothorakalen Übergangsbereich sicher diagnostiziert werden. Bei pathologischem neurologischen Befund ohne Korrespondenz im CT ist eine kernspintomografische Untersuchung anzuschließen. Akzelerations-/ Dezelerationstraumen können beim jungen Sportler häufig zu Läsionen und Querschnittsläsionen führen. Besonders gefährdet ist der Patient, wenn zusätzlich ein Hypomochlion oder sonstige Hebelkraft von außen einwirkt. Klassisch ist hier der Bandencheck beim Eishockey. Eine weitere klassische Verletzungsart, oft verbunden mit einer Querschnittsläsion, ist der Hechtsprung in die Untiefe. Hierzu darf aber nicht unerwähnt bleiben, dass die zugehörige „sportliche Herausforderung“ oft im Überschwang und unter Alkoholeinfluss erfolgt. Die operative Versorgung der Instabilität erfolgt klassisch über einen ventralen Zugang unter Ausräumen der verletzten Bandscheibe und Fusion mittels ventraler Platte. Als Bandscheiben-

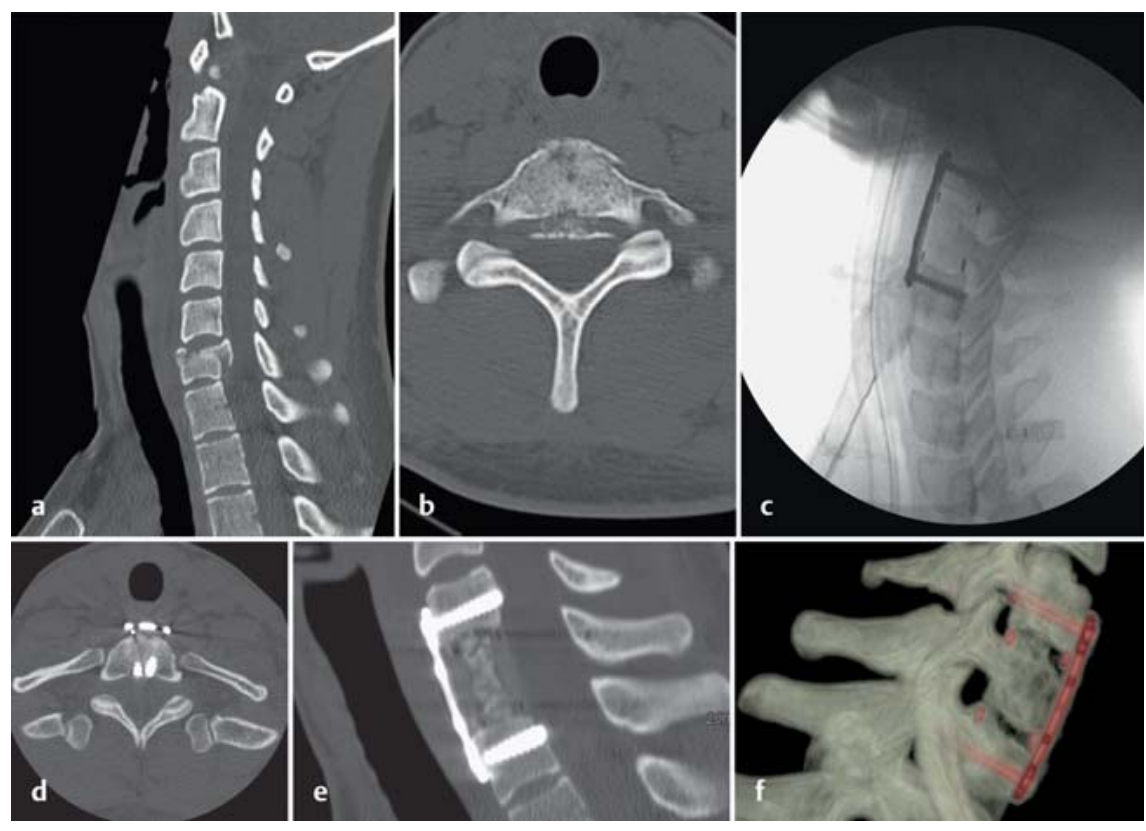

Abb. 1 a bis $\mathbf{f}$ 23-jährige Sportlerin mit C7-Luxationsfraktur und Neurologie (a sagittale 2DRekonstruktion, b CT-Schicht). Sofortige operative Versorgung mit Ausräumung des betroffenen Wirbelkörpers und Einsetzen eines Cages als Wirbelkörperersatz (c). Postoperative CTKontrollen zeigen den freien Spinalkanal und die korrekte Lage der Implantate (d CT-Schicht, e sagittale 2D-CT-Rekonstruktion, f 3D-Rekonstruktion).

ersatz fungiert regelhaft ein Beckenkammspan als autologe Knochenplastik, wobei hier zunehmend auch Cages aus Titan oder PEEK zum Einsatz kommen. Bei Luxationen gelingt in aller Regel die Reposition unter kontinuierlichem Zug, sodass die alleinige ventrale Versorgung ausreichend ist. Nur in Ausnahmefällen muss offen dorsal reponiert und stabilisiert und dann ventral (ein- oder zweizeitig) reponiert werden (Abb.1).

\section{BWS}

Zu unterscheiden ist der obere und mittlere Abschnitt vom thorakolumbalen Übergang. Letzterer stellt die zahlenmäßig weitaus bedeutendste Region dar und wird weiter unten ausführlich beschrieben.

Der obere und mittlere Abschnitt der BWS ist gekennzeichnet von einem sehr engen Spinalkanal in Relation zum Myelon. Es resultiert eine hohe Querschnittsgefährdung sowohl durch das Trauma als auch durch jegliche iatrogene Manipulation. Viele Verletzungen können konservativ behandelt werden, $\mathrm{zu}$ berücksichtigen ist allerdings gerade beim Sportler der hohe Anspruch an eine frühe Belastbarkeit. Flexions-/Distraktionsverletzungen im Sinne von B-Frakturen treten bei Sportverletzungen relativ häufig auf und sind regelhaft operativ zu versorgen. Die dorsale, dann meist multisegmentale Instrumentierung kann ausreichend sein, man muss allerdings mit einer gewissen Nachsinterung rechnen. Zum Erzielen einer frühen hohen Belastbarkeit der ventralen Säule ist die ventrale Abstützung vorteilhaft. Aufgrund der relativ klein dimensionierten Wirbelkörper und der hohen Vulnerabilität des Myeloms in diesen Abschnitten werden von den meisten Operateuren Beckenkammspäne bevorzugt verwendet, zunehmend kommen aber auch expandierbare Cages zum Einsatz. Die endoskopischen Techniken ermöglichen eine perfekte Übersicht bei geringer Morbidität. Aufgrund der anatomischen Lage ist der Zugang von rechts zu wählen (Abb. 2).

\section{Thorakolumbaler Übergang/LWS}

Das Therapiekonzept in dieser Region ist unabhängig vom Unfallmechanismus oder der ausgeführten Sportart und richtet sich nach dem in unserer Klinik maßgeblichen Behandlungs-Algorithmus, der hier kurz aufzuführen ist (Tab.1). Der Verletzungstyp wird anhand der präoperativen CT-Diagnostik nach AO klassifiziert. Das MRT kann ggfs. zur Differenzierung zwischen Aund B-Verletzungen herangezogen werden. Trotz aller moderner Diagnostik ist in manchen Fällen aufgrund der im Rah- 

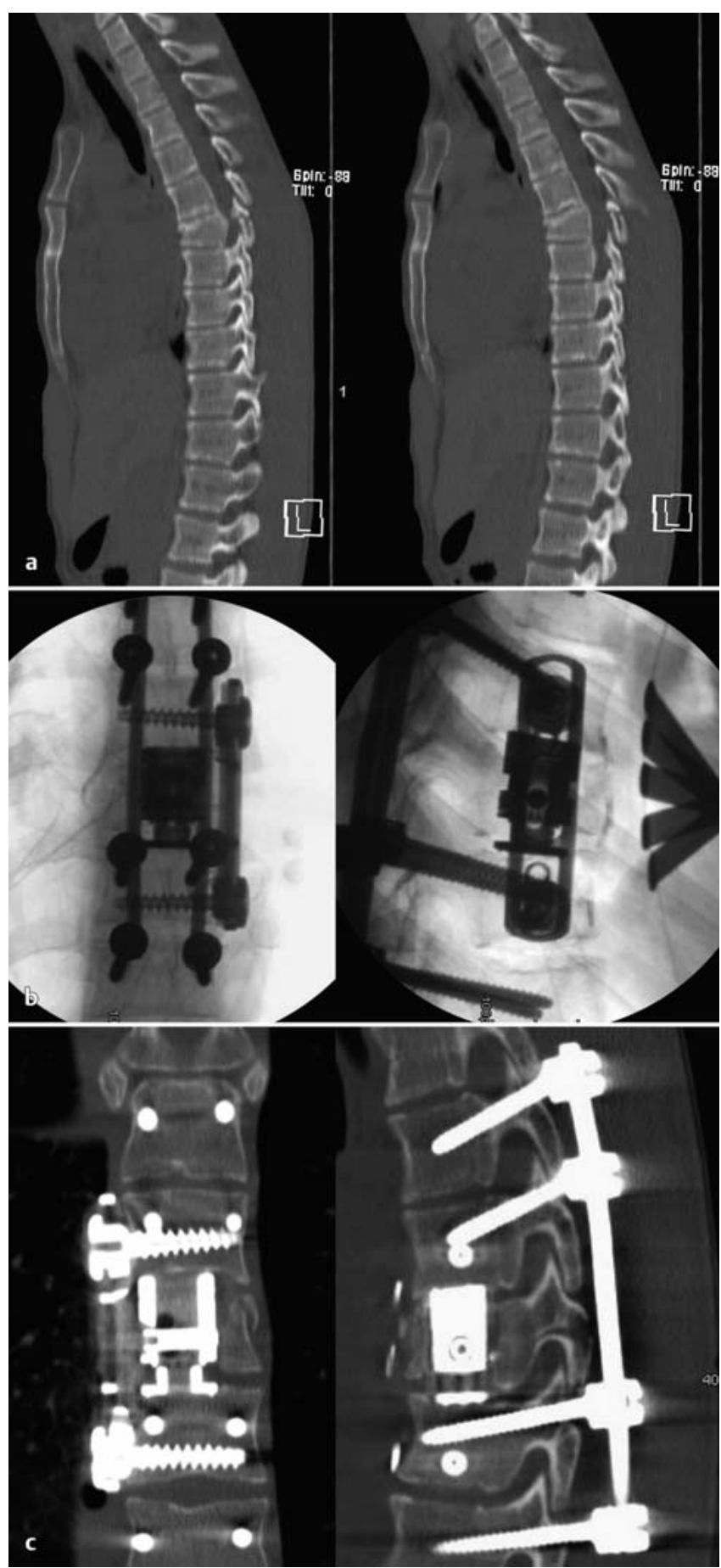

Abb. 2 a bis $\mathbf{c}$

Ein 17-jähriger Skispringer stürzt beim Übungssprung schwer, er zieht sich einen Berstungsbruch auf Höhe Th7 zu, mit dorsaler Komponente im Sinne einer B-Verletzung. Die primäre CT-Diagnostik erfolgt im erstbehandelnden Klinikum (a). Nach Verlegung in das Wirbelsäulenzentrum erfolgt die dorsoventrale Rekonstruktion und Stabilisierung mit multisegmentaler dorsaler Instrumentierung und expandierbarem Cage ventral. Intraoperative Verlaufskontrolle (b), postoperativer Verlauf in der frontalen und sagittalen 2D-Rekonstruktion des CT (c). men der dorsalen Instrumentierung erhobenen Befunde die Klassifikation zu korrigieren $[5,8]$.

Studiendesign: Im Rahmen einer prospektiven Studie wurden in unserer Klinik im 2-Jahres-Zeitraum 96 Patienten (69 Männer und 27 Frauen, Durchschnittsalter 35 Jahre) mit einer frischen Sportverletzung im Bereich von Brust- und/ oder Lendenwirbelsäule erfasst. Die Indikationsstellung zur operativen Versorgung erfolgte gemäß dem in unserer Klinik gültigen Algorithmus (Tab. 1). Ziel der operativen Versorgung an der Wirbelsäule war die Vermeidung von Fehlstellungen, Pseudarthrosen und Instabilität bei gleichzeitig geringer Eingriffsmorbidität zur Erzielung der möglichst frühen vollen Belastungsfähigkeit der Wirbelsäule und Rückkehr zur vollen Sportfähigkeit.

Für die dorsale Aufrichtung und Stabilisierung wurde im Bereich der unteren Brustwirbelsäule, des thorakolumbalen Übergangs und der Lendenwirbelsäule das Universal Spine System ${ }^{\circledR}$ (USS) der Fa. Synthes ${ }^{\circledR}$ verwendet. Bei Verletzungen im Bereich der oberen und mittleren Brustwirbelsäule kam das USS-Pedikelschraubensystem oder das USS-Pediatric-System ${ }^{\circledR}$ in der bekannten Standardtechnik zum Einsatz. Die ventrale Versorgung erfolgte in der an unserer Klinik entwickelten endoskopisch assistierten Operationstechnik [2,7], unter Verwendung eines winkelstabilen Implantates $\left(\mathrm{MACS} \mathrm{TL}^{\circledR}\right)[3,4]$. Als Wirbelkörperersatz dienten expandierbare Cages, wobei verschiedene Systeme zum Einsatz kamen. Beim Zugang zur mittleren bis unteren Lendenwirbelsäule wurde die Mini-Lumbotomietechnik unter Verwendung eines Synframe ${ }^{{ }}$Retraktorsystems eingesetzt.

Ergebnisse: Von 364 im Rahmen eines 2-Jahres-Zeitraumes an der Berufsgenossenschaftlichen Unfallklinik Murnau prospektiv erfassten Patienten mit Verletzungen der Brust- und Lendenwirbelsäule erlitten 96 Patienten ihre Verletzung beim Sport. Dies bedeutet immerhin einen Anteil von knapp 25\%!

Tab. 1 Algorithmus zur operativen Versorgung instabiler Verletzungen von Brust- und Lendenwirbelsäule [10].

\begin{tabular}{lcc}
\hline Isoliert dorsale Stabilisierung & Isoliert ventrale Stabilisierung & Kombinierte dorsoventrale Versorgung \\
\hline - Typ A3, B, C nach AO & - Typ A2.2-A3 nach AO & - Typ A3, B, C nach AO \\
- einfaches sagittales Verletzungsmuster & - gute Knochenqualität & - Höhenminderung des frakturierten \\
(Flexion/Extension) & - geringe posttraumatische Fehlstellung & Wirbelkörpers $>50 \%$ \\
- Integrität der angrenzenden Band- & - Höhenminderung $<50 \%$ des & - posttraumatische Fehlstellung im betrof- \\
scheibe & Wirbelkörpers & fenen Segment $>30^{\circ}$ \\
- geringer knöcherner Substanzdefekt & - posttraumatische Fehlstellung $<30^{\circ}$ & - großes Hinterkantenfragment \\
des Wirbelkörpers nach erfolgter & & - relevante Einengung des Spinalkanals \\
Aufrichtung & & - Zerreißung der angrenzenden Band- \\
& & scheiben
\end{tabular}


Nach der AO-Klassifikation lagen zu $60 \%$ A-, zu $18 \%$ B- und zu $22 \%$ C-Verletzungen vor, die häufigste Subklassifikation betraf den inkompletten Kompressionsbruch (A 3.1) mit 21\% Anteil. Bei $85 \%$ der Patienten bestand kein neurologisches Defizit (Frankel E), bei $4 \%$ zeigte sich eine komplette Neurologie (Frankel A). Frankel B, C oder D fanden sich in $11 \%$. Die Verletzungshöhe zeigte die übliche Verteilung wie sie aus der Literatur bekannt ist, in $67 \%$ lag sie im thorakolumbalen Übergangsbereich. Unter den Sportarten dominierten Wintersportarten wie Skifahren, Rodeln und Snowboarden. Aber auch Gleitschirmfliegen, Mountainbiken und Reiten sind zahlreich vertreten (Tab. 2). $19 \%$ der Patienten wurden konservativ behandelt. 78 Sportler wurden operiert, 17 isoliert dorsal, 11 isoliert ventral und 49 dorsoventral. Es traten 6 Komplikationen auf, einmal intraoperativ, wobei eine Segmentgefäß-Blutung ohne Konvertierung zum offenen Verfahren erfolgreich gestillt werden konnte. Weiterhin traten 3-mal ein punktionswürdiger postoperativer Erguss nach ventralem Eingriff, ein Infektverlauf im dorsalen Zugangsbereich nach isoliert dorsaler Versorgung und eine Spanpseudarthrose nach Verwendung eines Beckenkammspanes bei isoliert ventralem Vorgehen auf.

Insgesamt konnten $91 \%$ der verletzten Sportler weiter Sport betreiben, davon $84 \%$ mit der gleichen Häufigkeit und $68 \%$ mit der gleichen Intensität. Die Unfallsportart aufgehört haben $11 \%$ (hierunter allein 6 Gleitschirmflieger) und keinen Sport mehr seit dem Unfall betreiben $9 \%$ (davon 6 Patienten aufgrund der seit dem Unfall bestehenden Neurologie, einer mit einem schweren Schädel-Hirn-Trauma und 2 aufgrund der Schmerzsymptomatik) [10].

Bei der Entstehung von Wirbelsäulenverletzungen im Rahmen des Sports spielt die Zunahme von Risiko- und Hochrasanzsportarten eine nicht unerhebliche Rolle. Die technische Verbesserung z.B. von Wintersportgeräten mit Integration von Sicherheitsmodulen wie beispielsweise der Skibindungen mit einstellbaren Auslösemechanismen haben dazu beigetragen, den Skisport sicherer zu machen. Andererseits vermitteln sie auch ein falsches Sicherheitsgefühl, das die Risikobereitschaft fördert und $\mathrm{zu}$ höheren Geschwindigkeiten führt [9]. Die hierbei auftretenden hohen Beschleunigungskräfte schlagen sich auch im Verletzungsmuster nieder. So findet sich in den letzten Jahren eine Zunahme von schweren Verletzungen bei Ski- und Snowboardfahrern [13], einschließlich der Zunahme von
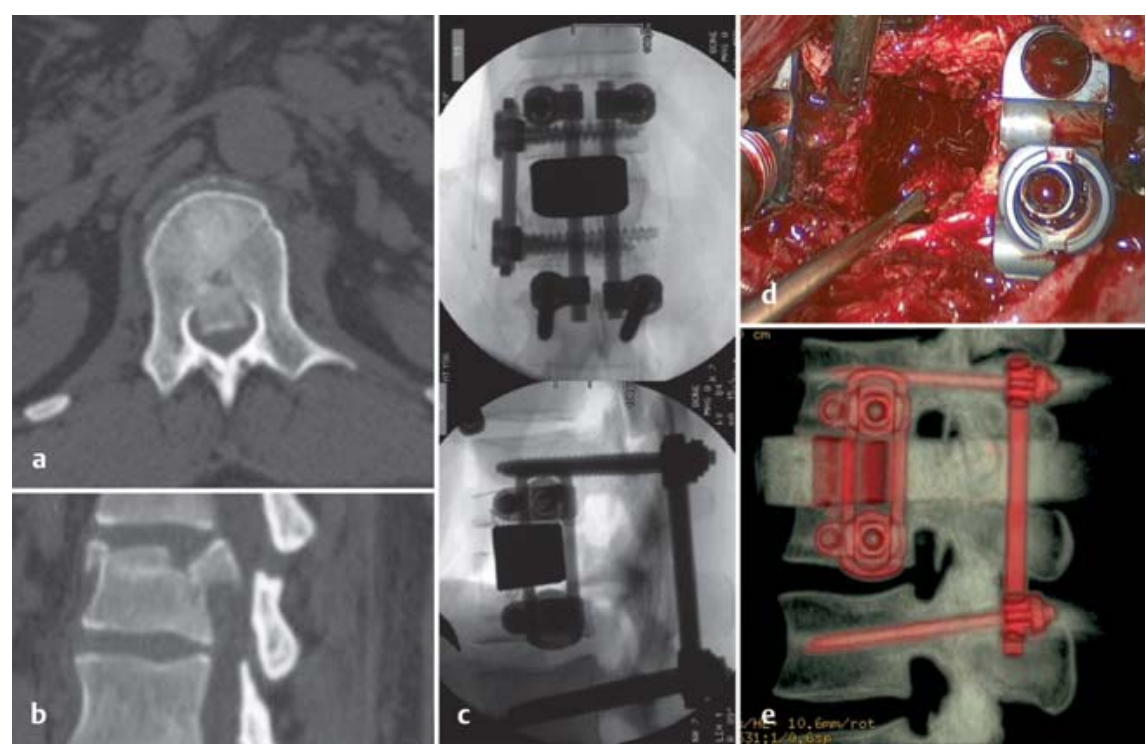

Abb. 3 a bis e Sportunfall mit A3.1-Verletzung auf Höhe Th12, im primären CT (a Schicht, b sagittale 2D-Rekonstruktion) $>50 \%$ Spinalkanaleinengung, klinisch fragliche Neurologie im Sinne einer Konus-Symptomatik. c Dorsoventrale Instrumentierung mit Hinterkantenresektion zum Spinalkanalclearing endoskopisch gestützt (d). Ventral erfolgt die monosegmentale Versorgung mit Tantalum-Cage, die dorsale bisegmentale Instrumentierung wird binnen 9 Monaten entfernt, um die unverletzte Bandscheibe T12/L1 bei dem engagierten Sportler wieder freizugeben. Die postoperative 3D-CT-Kontrolle zeigt die korrekte Lage der Implantate und die erfolgreiche Resektion des Hinterkantensegments (e).

Tab. 2 Häufigkeit der einzelnen Sportarten als Verletzungsursache bei Wirbelkörperfrakturen im eigenen Patientengut.

\begin{tabular}{ll} 
Verletzungsursache & Anteil (\%) \\
\hline Skifahren & 23 \\
\hline Gleitschirme & 18 \\
\hline Rodeln & 14 \\
\hline Snowboard & 11 \\
\hline Mountainbike & 9 \\
\hline Reiten & 8 \\
\hline Klettern & 4 \\
\hline Bergsteigen & 4 \\
\hline Wassersprung & 4 \\
\hline sonstiges & 5
\end{tabular}

Kopf- und Wirbelsäulenverletzungen [9,12]. Im Wintersport liegen die Wirbelsäulenverletzungen mit $7,5 \%$ bei 1000 Tagen nach den Kopf- und Abdominaltraumen an dritter Stelle $[9,15]$.

Einer Aufstellung von Keene [11] zufolge machen thorakolumbale Frakturen 5\% der Verletzungen im Skisport und 8\% der im Freestyle-Skifahren erlittenen Verletzungen aus. Nicht wenige der Wirbelsäulenverletzungen im Sport gehen mit einem neurologischen Defizit einher. In einer Analyse von 2346 querschnittgelähmten Patienten wurde ein Anteil von 8,3\% (194) gefunden, der auf Unfälle beim Sport und Baden zurückgeführt wurde. Insgesamt liegt in 17 bis $36 \%$ bei Ski- und Snowboardfahrern mit Wirbelsäulenverletzung ein neurologisches Defizit vor, wobei die Halswirbelsäulenverletzungen am häufigsten mit einer Neurologie einhergehen und Snowboardfahrer seltener als Skifahrer betroffen sind $[9,14]$.

Die Ausgangssituation von sportlich aktiven Individuen vor dem Unfall unterscheidet sich in mancherlei Hinsicht von der anderer Unfallverletzter. Vorteilhaft für den Erfolg konservativer wie operativer Behandlungsmaßnahmen ist das durchschnittlich junge Lebensalter. Ein überdurchschnittlich ausgebildetes Körpergefühl und Koordinationsvermögen, Disziplin und Frustrationstoleranz sind gute Voraussetzungen für eine hohe Akzeptanz der rehabilitativen Maßnahmen. Andererseits werden von diesen Patienten hohe Anforderungen an das Behandlungsergebnis gestellt, von dem erwartet wird, dass es in den allermeisten Fällen eine Wiederaufnahme der sportlichen Aktivität ermöglicht, die weit über dem durchschnittlichen Maß liegen. Diesem Umstand als auch 
den Anforderungen an die funktionelle Wiederherstellung wird man heute durch entsprechende moderne Operationsverfahren wie z.B. die minimalinvasive endoskopisch gestützte ventrale Spondylodese sowie dem Einsatz optimierter und hoch technisierter Implantate wie z.B. expandierbare Cages als Wirbelkörperersatz gerecht (Abb. 3).

\section{Schlussfolgerung}

Das Ziel der Behandlung von Wirbelsäulenverletzungen bei Sportlern ist die anatomische Wiederherstellung des Wirbelsäulenprofils und die Belastungsfähigkeit bei möglichst geringer Funktionsbeeinträchtigung. Durch den Einsatz minimalinvasiver Operationstechniken kann diesen Anforderungen weitestgehend entsprochen werden, sodass auch nach schweren Verletzungen der Wirbelsäule im Sport eine Rückkehr in die sportliche Aktivität in den meisten Fällen möglich ist. Durch adäquate Indikationsstellungen für die jeweiligen konservativen und operativen Versorgungsstrategien können durch beide Therapieansätze gute funktionelle Ergebnisse erzielt werden. Dennoch muss darauf hingewiesen werden, dass signifikante Unterschiede in Bezug auf den Korrekturverlust bei den einzelnen Verfahren resultieren. Dorsoventrale Versorgungen wiesen den geringsten Korrekturverlust auf, während konservativ und rein dorsal versorgte Verletzungen den höchsten Korrekturverlust im Outcome aufwiesen. Aus diesem Grunde erscheinen uns Studien mit Langzeit- ergebnissen von operativ und konservativ versorgten Patienten nach Wirbelsäulenverletzungen (auch oder gerade nach Sportverletzungen) besonders wichtig. Ggfs. wird im weiteren Verlauf eine Bestätigung oder Neueinschätzung der Versorgungsstrategie und Indikationsstellung erforderlich werden.

\section{Literatur}

1 Bailes Jr. JE, Van der Veer CA. Surgical management of patients with sports-related spinal injuries. Clin Neurosurg 2001; 48: 243 259

2 Beisse R, Muckley T, Schmidt MH, Hauschild M, Buhren V. Surgical technique and results of endoscopic anterior spinal canal decompression. J Neurosurg Spine 2005; 2: 128 - 136

${ }^{3}$ Böttner F. Facharztkompendium Orthopädie und Unfallchirurgie. 3. Aufl. Berlin: OrthoForum-Verlag; 2008: 249-267

${ }^{4}$ Briem D, Windolf J, Lehmann W, Begemann PG, Meenen NM, Rueger JM, Linhart W. Endoskopische Knochentransplantation an der Wirbelsäule: Ergebnisse der anterioren Fusion und therapeutische Konsequenzen. Unfallchirurg 2004; 107: 1152 - 1161

5 Josten C, Katscher S, Gonschorek O. Therapiekonzepte bei Frakturen des thorakolumbalen Überganges und der Lendenwirbelsäule. Orthopäde 2005; 34: 1021 - 1032

${ }^{6}$ Keene JS. Thoracolumbar fractures in winter sports. Clin Orthop Relat Res 1987; 216: 39 49

7 Kim DH, Jahng TA, Balabhadra RS, Potulski M, Beisse $R$. Thoracoscopic transdiaphragmatic approach to thoracolumbar junction fractures. Spine J 2004; 4: $317-328$

${ }^{8}$ Knop C, Blauth M, Bühren V, Hax P-M, Kinzl L, Mutschler W, Pommer A, Ulrich C, Wagner S, Weckbach A, Wetzensen A, Wörsdörfer O. Operative treatment of thoracolumbar fractures - part 3: follow-up. Results of prospektive multicenter study by the working group "spine" of the German Society of Trauma surgery. Unfallchirurg 2001; 104: 583-600
${ }^{9}$ Levy A, Smith $R$. Neurologic injuries in skiers and snowboarders. Semin Neurol 2000; 20: $233-245$

10 Merkel P, Hauck S, Zentz F, Bühren V, Beisse R. Wirbelsäulenverletzungen im Sport: Versorgungsstrategie und klinische Ergebnisse. Unfallchirurg [im Druck]

11 Micheli LJ. Sports following spinal surgery in the young athlete. Clin Orthop Relat Res 1985; 198: $152-157$

12 Prall J, Winston K, Brennan R. Severe snowboarding injuries. Injury 1995; 26: 539-542

13 Prall J, Winston K, Brennan R. Spine and spinal cord injuries in downhill skiers. J Trauma 1995; 39: 1115 - 1118

14 Silver JR. Spinal injuries in sports in the UK. Br J Sports Med 1993; 27: 115-120

15 Tarazi F, Dvorak M, Wing P. Spinal injuries in skiers and snowboarders. Am J Sports Med 1999; 27: 177 - 180

Dr. med. Oliver Gonschorek Leitender Arzt, Leiter der Wirbelsäulenchirurgie

Dr. med. Patrizia Merkel

Assistenzärztin

Dr. med. Michael Maier

Assistenzarzt

Dr. med. Stefan Hauck

Oberarzt

Prof. Dr. med. Volker Bühren

Ärztlicher Direktor

Berufsgenossenschaftliche

Unfallklinik Murnau

Prof.-Küntscher-Straße 8

82418 Murnau 